\title{
Bulletin to publish timely outbreak info
}

A fter languishing for five years, the federal government's public health information bulletin publicizing outbreak reports and other communicable disease news has been revamped as a pilot project to provide "practical and timely" information.

Some public health experts are pleased with this move; however, at least one cautions that resurrecting an old publishing model may not be the fastest way to get outbreak information to those who need it.

Canada Communicable Disease Report (CCDR), the fortnightly bulletin from the Public Health Agency of Canada, stopped publishing communicable disease news in 2009 and has since only featured statements from the National Advisory Committee on Immunization, FluWatch data and reports from other national public health newsletters. Many public health investigators attributed $C C D R$ 's decline to federal cutbacks, but the actual reason is unknown. Whatever the reason, the result is that some Canadian investigators started publishing their findings elsewhere notably, in other national bulletins.

Then a Nov. 29, 2013, editorial from Dr. Patricia Huston, $C C D R$ 's new scientific editor, announced it was being "revitalized" to "provide practical and timely information on infectious diseases in Canada for both public health and health care professionals."

Some have lauded this move, but Dr. Kumanan Wilson, Canada Research Chair in Public Health Policy at the University of Ottawa, says that for "emergent reporting, publication is a bit of an antiquated model." Real-time data updating for FluWatch and open-data initiatives, such as the Canadian Chronic Disease Surveillance System, are "the way the world is headed."

Although it would be "nice to have a Canadian journal" publishing analyses of the data, "the reality is people want to publish in the highest impact journals," which suggests that the need for a Canadian public health bulletin

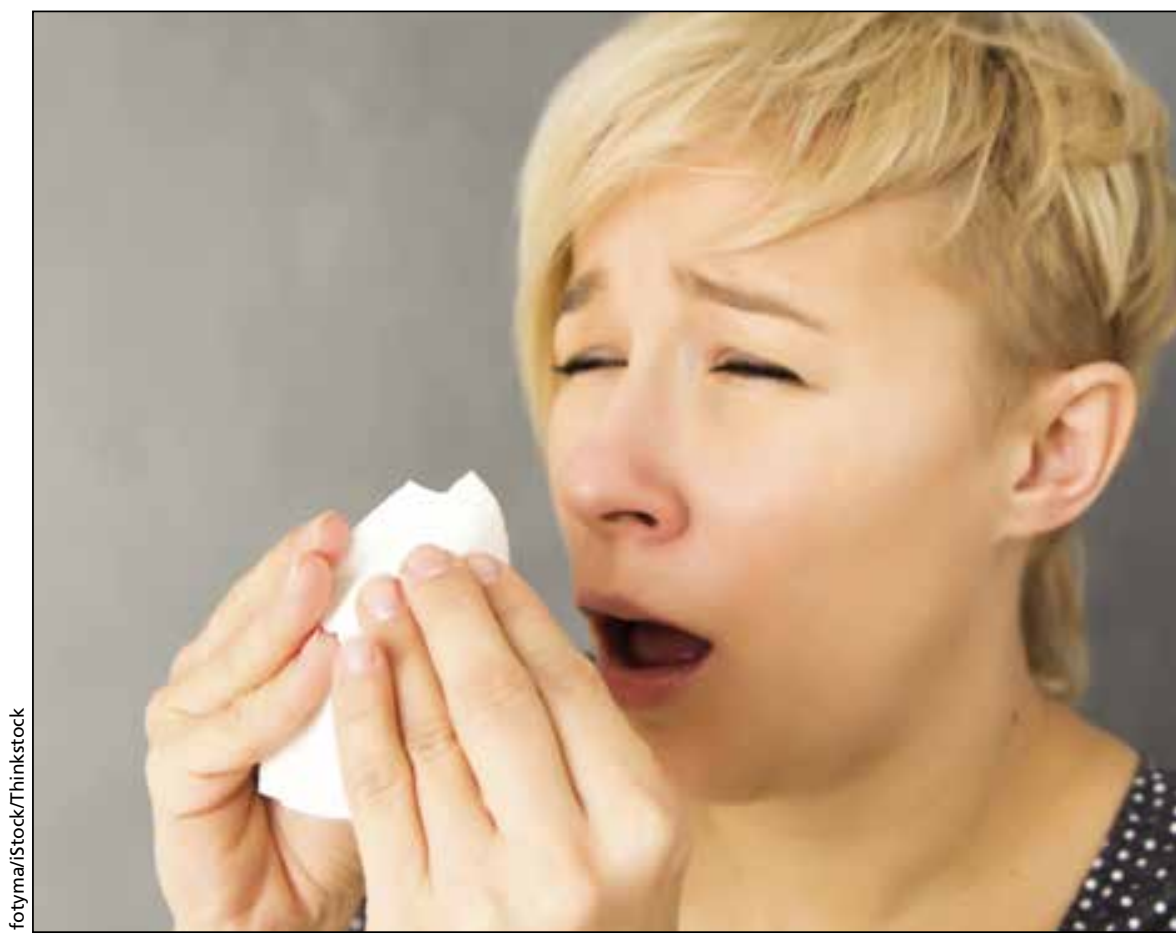

The Canada Communicable Disease Report recently resumed publishing communicable disease news, but some question the old-school media.

may not be as great as it once was, Wilson added.

That thinking may be a little ahead of its time, says Dr. Bonnie Henry, interim executive medical director of the British Columbia Centre for Disease Control in Vancouver. There is still pressure for academics to publish, and many public health workers hold academic appointments, says Henry. "There's a lot of support for a good venue for publishing practical public health information and we're really hopeful that $C C D R$ is going to be able to move in that direction." The "vision [for $C C D R$ ] is still being formed," she added.

"We've long lamented the demise of $C C D R$, so I'm actually really, really pleased" about its return, says Henry, who recently published information about a 2012 hepatitis A outbreak in $\mathrm{BC}$ in Eurosurveillance.

Dr. Danuta Skowronski, the BC centre's epidemiology lead for influenza and emerging respiratory pathogens, also supports more timely dissemination of information and a Canadian home for "summarizing and synthesizing key findings from Canadian investigations, surveillance and studies."

"There are other places that people can access information but that doesn't mean we don't also need [the CCDR]."

For the last three flu seasons, Skowronski and members of Canada's sentinel surveillance network have published interim estimates of flu vaccine effectiveness in Eurosurveillance. The European bulletin was able to accommodate their requirements of timeliness, peer review and citation in PubMed which even the current incarnation of $C C D R$ wasn't able to meet, Skowronski said. $C C D R$ would have taken three months; Eurosurveillance published their papers, with peer review, within 10 to 14 days of submission, she said.

"When you're talking about unfolding outbreak news or mid-season vaccine effectiveness reporting, that is the kind of standard that we're seeking," Skowronski said. 
"A regular consolidated home journal summarizes and interprets key findings, actively distributes/notifies recipients, is subsequently searchable and can be referenced by others. And, in the case of MMWR (Morbidity and Mortality Weekly Report, the weekly bulletin of the US Centers for Disease Control and Prevention [CDC]) or Eurosurveillance, it can accommodate real-time notification and distribution electronically when needed," Skowronski added.

"In other words, agency website postings and a national journal need not be mutually exclusive. The CDC and the European Centre for Disease Prevention and Control use both options already. Why shouldn't Canada?"

But Jana Lerner, a spokesperson for the federal agency told CMAJ that the $C C D R$ 's renewal is a "pilot project," and it's not clear how long the new bulletin will run.

The Public Health Agency of Canada would not make Huston or anyone else affiliated with $C C D R$ available for an interview. Instead, the spokesperson stated that $C C D R$ has been "reinstated as a pilot project as the agency explores different approaches for knowledge translation on infectious diseases to clinicians, public-health professionals, and policy-makers.

"The goal of the Agency is to disseminate timely information on current and emerging infectious diseases in Canada, and we are exploring if $C C D R$ is the best tool available for this purpose." - Terry Murray, Toronto, Ont.

CMAJ 2014. DOI:10.1503/cmaj.109-4836 